\title{
SODIUM NITROPRUSSIDE IN PATIENTS WITH COMPROMISED LEFT VENTRICULAR FUNCTION UNDERGOING CORONARY BYPASS: REDUCTION OF CARDIAC PROINFLAMMATORY SUBSTANCES
}

Parwis Massoudy, MD

Stefan Zahler, $\mathrm{PhD}^{\mathrm{b}}$

Tobias Freyholdt ${ }^{c}$

Richard Henze, MD

Andreas Barankay, $\mathrm{MD}^{\mathrm{d}}$

Bernhard F. Becker, MD, $\mathrm{PhD}^{\mathrm{b}}$

Siegmund L. Braun, MD

Hans Meisner, $\mathrm{MD}^{\mathrm{c}}$
Objective: The aim of the present study was to investigate whether the nitric oxide donor sodium nitroprusside can reduce the cardiac inflammatory response during coronary artery bypass grafting in patients with severely compromised left ventricular function. Methods: Patients $(n=30)$ were assigned to receive placebo or sodium nitroprusside $(0.5$ $\mu \mathrm{g} \cdot \mathrm{kg}^{-1} \cdot \mathrm{min}^{-1}$ ) for the first 60 minutes of reperfusion. Interleukin 6 , interleukin 8, and tumor necrosis factor $\alpha$ levels; platelet adhesion molecule CD41 and CD62 levels; and CD11b on leukocytes were determined in the radial artery and coronary sinus before cardiopulmonary bypass and during reperfusion $(1,5,10,35$, and 75 minutes). Results: At 1 minute of reperfusion, coronary venous levels of CD41-positive polymorphonuclear leukocytes were $8 \%$ lower than arterial levels in the placebo group and $18 \%$ higher in the sodium nitroprusside group $(P=$ $.021)$. At 5 minutes of reperfusion, the respective levels were $29 \%$ and $1 \%$ for interleukin $6(P=.015),-5 \%$ and $20 \%$ for CD41-positive monocytes $(P=.032)$, and $-2 \%$ and $16 \%$ for CD11b-positive monocytes $(P=$ .038). At 10 minutes of reperfusion, these levels were $-14 \%$ and $21 \%$ for CD41-positive monocytes $(P=.006)$. At 35 minutes of reperfusion, these levels were $-\mathbf{1 3 \%}$ and $7 \%$ for CD41-positive monocytes $(P=.017),-41 \%$ and $23 \%$ for CD11b-positive monocytes $(P=.001)$, and $7 \%$ and $25 \%$ for CD62-positive platelets $(P=.041)$. At 75 minutes of reperfusion, the levels were $15 \%$ and $-7 \%$ for tumor necrosis factor $\alpha(P=.025)$ and $-\mathbf{1 0 \%}$ and $10 \%$ for CD62-positive platelets $(P=.041)$. Conclusions: Transcardiac production of proinflammatory cytokines is reduced in patients undergoing coronary artery bypass grafting treated with the nitric oxide donor sodium nitroprusside. At the same time, less activated leukocytes and platelets are retained in the coronary circulation. ( $J$ Thorac Cardiovasc Surg 2000;119:566-74)
$T^{1}$ he human heart has been shown to be a site of inflammation during postischemic reperfusion in patients undergoing coronary artery bypass grafting

From the Department of Cardiovascular Surgery, ${ }^{a}$ University of Essen, Essen, Germany; Department of Cardiovascular Surgery, ${ }^{c}$ Department of Anesthesiology, ${ }^{\mathrm{d}}$ and Department of Laboratory Medicine, ${ }^{\mathrm{e}}$ German Heart Center, Munich; and Department of

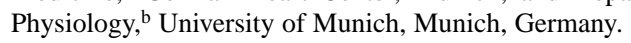

Supported by the Deutsche Forschungsgemeinschaft (MA 1731/3-1).

Received for publication March 22, 1999; revisions requested July 26, 1999; revisions received Oct 25, 1999; accepted for publication Oct 26, 1999.

Address for reprints: Parwis Massoudy, MD, Department of Cardiovascular Surgery, University of Essen, Hufelandstr 55, 45147 Essen, Germany (E-mail: parwis.massoudy@uni-essen.de).

Copyright (C) 2000 by Mosby, Inc.

0022-5223/2000 $\$ 12.00+0 \quad \mathbf{1 2 / 1 / 1 0 4 0 3 1}$

doi:10.1067/mtc. 2000.104031
(CABG). ${ }^{1} \mathrm{~A}$ transcardiac production of inflammatory substances has also been shown in patients treated with percutaneous transluminal coronary angioplasty (PTCA) for myocardial infarction. ${ }^{2}$

In experimental studies nitric oxide (NO) has been shown to reduce reperfusion injury. The concomitant cardioprotective effect of sodium nitroprusside (SNP) under experimental conditions was interpreted to result from radical scavenging by NO. ${ }^{3,4}$ In a clinical study in pediatric patients undergoing cardiac operations for congenital malformations, a reduction of complement activation during cardiopulmonary bypass (CPB) was observed with SNP. ${ }^{5}$ This was responsible for the diminished rise in inflammatory parameters noted by the authors. In patients with normal left ventricular function undergoing elective CABG, short-term (20 minutes) application of the NO donor SNP during 
Table I. Preoperative and intraoperative patient data

\begin{tabular}{|c|c|c|c|}
\hline & Placebo & $S N P$ & $\mathrm{P}$ value \\
\hline Patient age (y) & $63 \pm 7$ & $67 \pm 6$ & .05 \\
\hline Patient sex & 3 women, 12 men & 4 women, 11 men & $>.2$ \\
\hline Ejection fraction $(\%)$ & $33 \pm 10.2$ & $37 \pm 8.7$ & .06 \\
\hline Myocardial infarction & $1.1 \pm 0.7$ & $0.9 \pm 0.5$ & .2 \\
\hline NYHA class & $2.4 \pm 0.5$ & $2.4 \pm 0.5$ & $>.2$ \\
\hline Blood pressure at beginning of reperfusion $(\mathrm{mm} \mathrm{Hg})$ & $61 \pm 10$ & $56 \pm 14$ & .2 \\
\hline Decrease of blood pressure during reperfusion $(\mathrm{mm} \mathrm{Hg})$ & $24 \pm 11$ & $24 \pm 10$ & $>.2$ \\
\hline Duration of ischemia (min) & $65 \pm 13$ & $68 \pm 12$ & $>.2$ \\
\hline Duration of reperfusion (min) & $34 \pm 9$ & $31 \pm 9$ & $>.2$ \\
\hline No. of distal anastomoses & $3.4 \pm 0.7$ & $3.5 \pm 0.8$ & $>.2$ \\
\hline No. of proximal anastomoses & $1.9 \pm 0.7$ & $1.9 \pm 0.5$ & $>.2$ \\
\hline Dopamine after termination of $\mathrm{CPB}\left(\mu \mathrm{g} \cdot \mathrm{kg}^{-1} \cdot \mathrm{min}^{-1}\right)$ & $5.4 \pm 2.2$ & $5.7 \pm 1.8$ & $>.2$ \\
\hline Dobutamine after termination of CPB $\left(\mu \mathrm{g} \cdot \mathrm{kg}^{-1} \cdot \min ^{-1}\right)$ & $1.2 \pm 2.8$ & $1.7 \pm 2.4$ & $>.2$ \\
\hline Patients requiring norepinephrine after $\mathrm{CPB}$ & $2 / 15$ & $3 / 15$ & $>.2$ \\
\hline
\end{tabular}

Values are means $\pm \mathrm{SD}(\mathrm{n}=15$ patients each). The $\mathrm{Z}$ test was used for statistical comparison of patient sex and of patients requiring norepinephrine after $\mathrm{CPB}$. NYHA, New York Heart Association.

postischemic reperfusion was able to reduce the systemic inflammatory response reaction and also the transcardiac production of inflammatory mediators. ${ }^{6} \mathrm{~A}$ negative inotropic potential of cytokines has been described. ${ }^{7}$ Therefore the present study was performed in patients with severely compromised left ventricular function undergoing $\mathrm{CABG}$ to investigate whether the administration of SNP could mitigate the inflammatory response of the heart to $\mathrm{CPB}$ and ischemia-reperfusion and at the same time lead to less need for catecholamines after termination of CPB. Parameters measured included the proinflammatory cytokines interleukin 6 (IL-6), IL-8, and tumor necrosis factor $\alpha$ (TNF- $\alpha$ ); leukocyte adhesion molecule CD11b; and platelet adhesion molecules CD41 and CD62.

\section{Patients and methods}

Anesthesia. Anesthesia was conducted as a total intravenous technique. Induction of anesthesia was performed with $1 \mu \mathrm{g} / \mathrm{kg}$ sufentanil, $0.04 \mathrm{mg} / \mathrm{kg}$ midazolam, and 0.1 $\mathrm{mg} / \mathrm{kg}$ pancuronium. A loading dose of $3 \mu \mathrm{g} / \mathrm{kg}$ sufentanil and $0.1 \mathrm{mg} / \mathrm{kg}$ midazolam was given before sternotomy. Thereafter the infusion was adjusted to $0.5 \mu \mathrm{g} \cdot \mathrm{kg}^{-1} \cdot \mathrm{h}^{-1}$ sufentanil and $0.03 \mathrm{mg} \cdot \mathrm{kg}^{-1} \cdot \mathrm{h}^{-1}$ midazolam and maintained until the end of the operation. Dopamine $\left(3 \mu \mathrm{g} \cdot \mathrm{kg}^{-1} \cdot \mathrm{min}^{-1}\right)$ was used throughout the procedure to maintain adequate urine output. Before separation from $\mathrm{CPB}$, the infusion rate was set according to the patient's circulatory state. A cardiac index of at least $2.3 \mathrm{~L} \cdot \mathrm{min}^{-1} \cdot \mathrm{m}^{-2}$ and a mean arterial pressure of at least $60 \mathrm{~mm} \mathrm{Hg}$ were the targets. Aprotinin (Trasylol; Bayer, Leverkusen, Germany) was given according to the Hammersmith protocol (total dose 6 million KIU).

Operation and intervention. All patients underwent CABG with CPB by using roller pumps (Stöckert, Munich, Germany) and disposable membrane oxygenators (Dideco, Mirandola, Italy). The pump was primed with $1500 \mathrm{~mL}$ of lactated Ringer solution to which $100 \mathrm{mmol}$ of sodium bicarbonate and $5000 \mathrm{IU}$ of heparin (Ratiopharm, Ulm, Germany) were added. CPB was instituted at a flow rate of $2.4 \mathrm{~L} \cdot \mathrm{min}^{-1}$ $\cdot \mathrm{m}^{-2}$ body surface area after systemic heparinization (1 $\mathrm{mg} / \mathrm{kg}$ ). After the application of heparin, an activated clotting time of greater than 400 seconds was the target. When aprotinin was added, the target became 700 seconds. The body temperature was cooled to $26^{\circ} \mathrm{C}$ to $28^{\circ} \mathrm{C}$ (moderate hypothermia), and $1000 \mathrm{~mL}$ of cold Bretschneider solution (Custodiol; Köhler Chemie, Alsbach-Hähnlein, Germany) was applied as antegrade cardioplegia after clamping of the aorta. The remaining blood of the $\mathrm{CPB}$ circuit was prepared by using a Cell-Saver device (Haemonetics, Munich, Germany) before retransfusion.

After release of the aortic crossclamp, patients received either 5\% glucose solution (the solvent for Nipruss) as placebo or SNP (Nipruss $0.5 \mu \mathrm{g} \cdot \mathrm{kg}^{-1} \cdot \mathrm{min}^{-1}$ ), both of which were infused through the central venous line of a Swan-Ganz catheter (Baxter Healthcare Corp, Edwards Division, Santa Ana, Calif). The dose was chosen according to pre-experiments, in which $0.5 \mu \mathrm{g} \cdot \mathrm{kg}^{-1} \cdot \mathrm{min}^{-1}$ was the highest dose that showed no significant hemodynamic effects. The infusion was stopped after 60 minutes. Protamine $(1 \mathrm{mg} / \mathrm{kg})$ was given after CPB.

The use of cardiotomy suction was reduced to a minimum while proximal anastomoses were completed. Cardiotomy suction was not used after termination of CPB.

Blood samples. The blood for arterial measurements was drawn from a cannula in the radial artery. The coronary venous blood was withdrawn from a catheter (multipurpose, 6F; Cordis; Roden, The Netherlands), which was transcutaneously placed into the coronary sinus through the internal jugular vein under fluoroscopic control. The correct position of this catheter was monitored by registering the pressure curve and determining oxygen saturation (25\%-45\%) of samples from coronary sinus blood. Samples were taken at the following time points: (1) after application of heparin; (2) 1, 
Table II. Radial artery values for the cytokines $I L-6, I L-8$, and $T N F-\alpha$, as well as the adhesion molecules CDIIb on monocytes and PMNs, CD41 and CD62 on platelets, and CD41 on monocytes and PMNs

\begin{tabular}{|c|c|c|c|c|c|c|}
\hline & Pre- $C P B$ & $1 \mathrm{~min}$ & $5 \min$ & $10 \mathrm{~min}$ & $35 \mathrm{~min}$ & $75 \min$ \\
\hline \multicolumn{7}{|l|}{ Placebo } \\
\hline IL-6 (pg/mL) & $15.9 \pm 5.3$ & $85.7 \pm 12.2$ & $140.3 \pm 20.7$ & $133.4 \pm 20.7$ & $320.3 \pm 46.3$ & $248.0 \pm 85.2$ \\
\hline IL-8 (pg/mL) & $6.0 \pm 0.4$ & $11.8 \pm 1.2$ & $12.0 \pm 1.1$ & $12.6 \pm 1.3$ & $21.4 \pm 2.3$ & $28.3 \pm 7.6$ \\
\hline TNF- $\alpha(\mathrm{pg} / \mathrm{mL})$ & $9.3 \pm 0.8$ & $11.3 \pm 1.1$ & $11.6 \pm 1.0$ & $12.0 \pm 0.8$ & $14.0 \pm 1.5$ & $25.4 \pm 9.4$ \\
\hline CD11b monocytes (fluorescence units) & $16.1 \pm 4.1$ & $17.9 \pm 2.8$ & $18.6 \pm 2.9$ & $16.5 \pm 2.5$ & $17.3 \pm 3.9$ & $11.8 \pm 1.8$ \\
\hline CD11b PMNs (fluorescence units) & $15.1 \pm 4.4$ & $13.6 \pm 2.6$ & $14.8 \pm 3.4$ & $12.5 \pm 2.2$ & $13.2 \pm 3.5$ & $12.2 \pm 2.2$ \\
\hline CD41 platelets (fluorescence units) & $94.5 \pm 10.9$ & $125.8 \pm 14.0$ & $129.8 \pm 13.8$ & $132.3 \pm 13.2$ & $120.5 \pm 13.6$ & $136.7 \pm 12.9$ \\
\hline CD62 platelets (fluorescence units) & $8.3 \pm 1.8$ & $12.6 \pm 2.8$ & $12.7 \pm 2.9$ & $13.2 \pm 2.9$ & $12.8 \pm 2.8$ & $15.7 \pm 2.7$ \\
\hline CD41 monocytes (\% positive) & $31.3 \pm 3.3$ & $43.5 \pm 5.7$ & $48.2 \pm 5.5$ & $45.2 \pm 5.6$ & $40.9 \pm 6.5$ & $37.6 \pm 5.1$ \\
\hline CD41 PMNs (\% positive) & $23.5 \pm 2.5$ & $23.1 \pm 2.9$ & $22.5 \pm 2.3$ & $20.7 \pm 2.3$ & $20.0 \pm 2.7$ & $19.2 \pm 2.4$ \\
\hline \multicolumn{7}{|l|}{ SNP } \\
\hline IL-6 (pg/mL) & $12.9 \pm 2.7$ & $49.8 \pm 9.4$ & $87.2 \pm 17.1$ & $105.8 \pm 20.3$ & $240.2 \pm 44.1$ & $340.9 \pm 46.6$ \\
\hline IL-8 (pg/mL) & $7.0 \pm 1.1$ & $17.9 \pm 4.7$ & $18.3 \pm 4.4$ & $19.3 \pm 4.2$ & $32.2 \pm 5.8^{*}$ & $33.6 \pm 6.6$ \\
\hline TNF- $\alpha(\mathrm{pg} / \mathrm{mL})$ & $9.3 \pm 1.7$ & $11.9 \pm 1.9$ & $12.2 \pm 1.7$ & $12.6 \pm 1.9$ & $16.9 \pm 2.8$ & $29.9 \pm 7.6$ \\
\hline CD11b monocytes (fluorescence units) & $13.8 \pm 1.4$ & $17.3 \pm 2.5$ & $19.2 \pm 3.9$ & $17.1 \pm 2.7$ & $15.2 \pm 2.4$ & $15.1 \pm 1.9$ \\
\hline CD11b PMNs (fluorescence units) & $12.9 \pm 1.4$ & $15.8 \pm 2.3$ & $19.7 \pm 5.1$ & $15.9 \pm 3.2$ & $12.6 \pm 1.4$ & $14.8 \pm 2.3$ \\
\hline CD41 platelets (fluorescence units) & $87.0 \pm 9.8$ & $117.9 \pm 12.5$ & $119.5 \pm 11.9$ & $115.9 \pm 12.1$ & $109.5 \pm 10.3$ & $121.2 \pm 12.4$ \\
\hline CD62 platelets (fluorescence units) & $10.0 \pm 1.9$ & $14.0 \pm 2.6$ & $15.4 \pm 2.5$ & $15.0 \pm 2.5$ & $14.4 \pm 2.4$ & $16.5 \pm 2.6$ \\
\hline CD41 monocytes (\% positive) & $37.8 \pm 4.4$ & $48.4 \pm 4.0$ & $46.5 \pm 3.7$ & $46.3 \pm 3.4$ & $44.6 \pm 3.3$ & $42.3 \pm 3.2$ \\
\hline CD41 PMNs (\% positive) & $26.0 \pm 2.5$ & $28.0 \pm 3.0$ & $24.9 \pm 2.2$ & $23.5 \pm 2.4$ & $23.3 \pm 2.4$ & $22.2 \pm 2.8$ \\
\hline
\end{tabular}

Values are means \pm SEM. The columns indicate time points of blood sampling before CPB followed by 5 time points during reperfusion $(\mathrm{n}=15$ patients each).

${ }^{*} P=.046$ versus placebo.

5, and 10 minutes after release of the aortic crossclamp; (3) after termination of CPB (approximately 35 minutes after release of the aortic crossclamp), and (4) 75 minutes after release of the aortic crossclamp. Blood was simultaneously drawn from the radial artery and the coronary sinus at all time points.

Patients. The study was performed in a prospective and double-blind fashion. Inclusion criteria were as follows: (1) a left ventricular ejection fraction of $40 \%$ or less, (2) age of 80 years or less, and (3) no myocardial infarction (as defined by significant elevation of serum creatine kinase levels and/or electrocardiographic signs for myocardial infarction) within 14 days before the operation.

Exclusion criteria were known hypersensitivity against SNP or any other nitro substances, inflammatory diseases, or the intake of immunosuppressive drugs. A further exclusion criterion was unstable angina. Only patients undergoing elective $\mathrm{CABG}$ were enrolled.

Every patient with a profile suitable for enrollment in the study was informed about the nature of the study and about potential side effects of the application of SNP and was required to sign a written consent form 1 day before the operation. The preoperative patient characteristics are shown in Table I. The study was approved by the local ethics committee in August 1996. SNP (Nipruss) was donated by Schwarz Pharma (Monheim, Germany). The investigation conforms with the principles outlined in the Declaration of Helsinki.

From November 1997 until December 1998, 58 patients were elected to take part in the study and had signed the consent form. However, 28 patients had to be excluded because the coronary sinus catheter, which was placed in the operating room, could not be positioned in the right place within the time limit set for this procedure (15 minutes).

Immunoassays. For analysis of cytokines and cell counts, blood specimens were collected into heparinized tubes (Sarstedt, Nümbrecht, Germany). Plasma samples were stored frozen at $-20^{\circ} \mathrm{C}$ until assayed within batches. IL-6, IL8 , and TNF- $\alpha$ levels were measured by using a solid-phase, 2-site, chemiluminescent enzyme immunometric assay (Immulite system; Diagnostic Products Corporation, Los Angeles, Calif). Cardiac release of IL-6, IL- 8 , and TNF- $\alpha$ was calculated from the respective coronary venoarterial differences at each time point.

Flow cytometry. Flow cytometry of adhesion molecules on platelets and leukocytes was performed as previously described. ${ }^{1}$ In brief, for determination of leukocyte adhesion molecules, aliquots of the blood samples were mixed with 1 $\mathrm{mL}$ of FACS lysing solution (Becton Dickinson, Heidelberg, Germany) and double stained with anti-CD41 antibodies (Serotec, Kidlington, Oxford, Great Britain) and anti-CD11b antibodies (Exalpha, Boston, Mass). For analysis of platelets, $100 \mu \mathrm{L}$ of blood and $1 \mathrm{~mL}$ of Cellfix (Becton Dickinson) were mixed and incubated with antibodies against CD41 (Serotec) and CD62 (Harlan Seralab, Crawley Down, Sussex, Great Britain).

Flow cytometry was performed with a FACScan and Lysis II software (Becton Dickinson). The mean fluorescence intensity was taken as a measure of antibody binding. The nonspecific background was quantified by measurement of the fluorescence intensity of samples labeled with nonbind- 
ing isotype-matched antibodies and subsequently subtracted. Results are given in the percentage increase or decrease of a parameter in the coronary venous sample compared with the respective arterial sample. This normalization was necessary because of the considerable scatter of the baseline levels of the individual patients.

Statistical analysis. All results are expressed as mean values \pm SEM. The Student $t$ test for paired samples was used to evaluate differences between the groups.

\section{Results}

Interleukins and adhesion molecules. Arterial values for the cytokines IL- 6 , IL- 8 , and TNF- $\alpha$, as well as the adhesion molecules $\mathrm{CD} 11 \mathrm{~b}$ on monocytes and polymorphonuclear leukocytes (PMNs), adhesion molecules CD41 and CD62 on platelets, and CD41 on monocytes and PMNs are shown in Table II. The arterial values are similar between the groups, with the exception of the IL-8 levels after termination of CPB. Here a higher value was measured in the SNP group, which cannot be readily explained.

In the case of the cytokines, there was a general trend toward lower concentrations in the coronary sinus. Coronary venoarterial differences of IL- 6 and TNF- $\alpha$ levels are shown in Figs 1 and 2 as percentage values because of interindividual scatter. In contrast to the placebo group, the transmyocardial difference for IL-6 in the SNP group remained near zero at all time points after the first-minute reperfusion.

Coronary venoarterial differences for TNF- $\alpha$ were similar in both groups in early reperfusion. However, in contrast to the SNP group, after 75 minutes of reperfusion, a production of TNF- $\alpha$ was observed in the placebo group (Fig 2).

The application of SNP exerted no significant effects on coronary venoarterial differences of IL-8.

The general trend in the transcardiac differences of activated leukocytes and platelets was toward a retention in the placebo group and toward a washout in the SNP group. This was observed in the case of CD11bpositive monocytes at 1 and 30 minutes of reperfusion (Fig 3).

No significant differences in transcardiac gradients of CD11b expression on PMNs or CD41 expression on platelets were observed at any time point.

Figs 4 and 5 show the coronary venoarterial differences for the expression of the platelet adhesion molecule CD41 on monocytes and PMNs, respectively. The above-mentioned trend was again present, with a washout of CD41-positive monocytes occurring in the SNP group and a retention in the placebo group (Figs 4 and 5).
The same general direction was present in the case of CD62-positive platelets (Fig 6).

Clinical characteristics. All 30 patients survived. Preoperative, intraoperative, and postoperative characteristics are shown in Table I. Decline in blood pressure was equal in patients receiving placebo and those receiving SNP. No significant differences could be observed concerning the intraoperative characteristics or the need for catecholamines (dopamine, dobutamine, and norepinephrine) after termination of CPB. There was no low output syndrome in either group and no need to use an intra-aortic balloon pump in any of the patients. Postoperative myocardial infarction was diagnosed when significant ST-segment elevation was present in the electrocardiogram and significant myocardial enzyme increase was determined (myocardial fraction of creatine kinase greater than $10 \%$ of systemic levels). In 2 of 15 patients receiving placebo, temporary ST-segment elevation was noted without significant myocardial creatine kinase levels. The same nonspecific changes were observed in 1 of 15 patients receiving SNP. The highest creatine kinase level in the placebo group was 899 IU/L, with a myocardial fraction of $25 \mathrm{IU} / \mathrm{L}$. This patient did not have electrocardiographic abnormalities. The highest creatine kinase level in the SNP group was $1080 \mathrm{IL} / \mathrm{L}$, with a myocardial fraction of $21 \mathrm{IU} / \mathrm{L}$. No electrocardiographic abnormalities were observed.

\section{Discussion}

In the present study the application of SNP to patients undergoing CABG with compromised left ventricular function for the first 60 minutes of reperfusion led to a reduction of the acute cardiac inflammatory response. In addition to diminished cardiac production of IL-6 and TNF- $\alpha$, a washout of platelet-bearing leukocytes and of activated platelets, as well as of activated leukocytes during coronary passage, was observed in SNP-treated patients.

The effect was achieved without inducing systemic hemodynamic changes with SNP that outranged those occurring in the placebo group. Theoretically, coronary vascular resistance and flow rate, which were not measured in this study, could have been altered by low-dose SNP. However, in the presence of pre-existing ischemic vasodilation, such an effect seems rather unlikely.

Interleukins and adhesion molecules. Cardiac cytokine release has been shown during recanalization by means of PTCA in acute myocardial infarction, along with a significant cardiac production of IL-6 during reperfusion of the heart undergoing infarction, ${ }^{2}$ in a previous study in patients undergoing $\mathrm{CABG}$ with nor- 


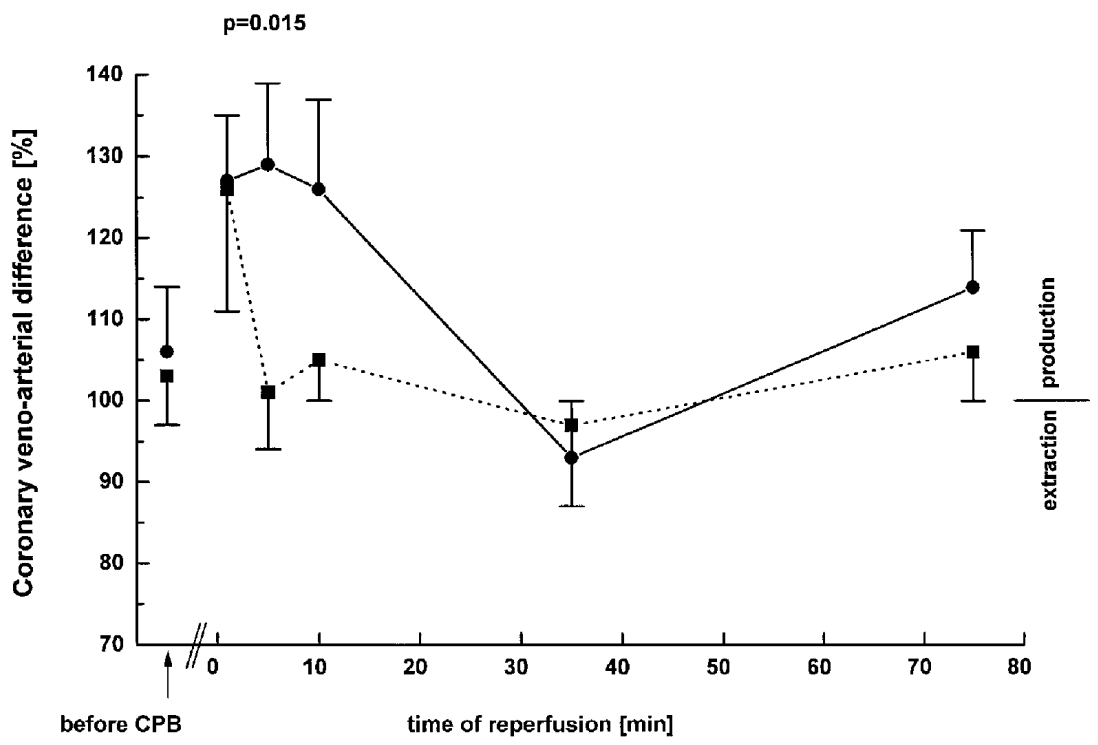

Fig 1. Coronary venoarterial difference of IL-6 levels, with the respective arterial value taken as $100 \%$. Values are means, and bars represent SEM ( $\mathrm{n}=15$ for each group; filled circles, placebo group patients; filled squares, SNP group patients). When differences between groups were significant, the respective levels are written on top of the symbols.

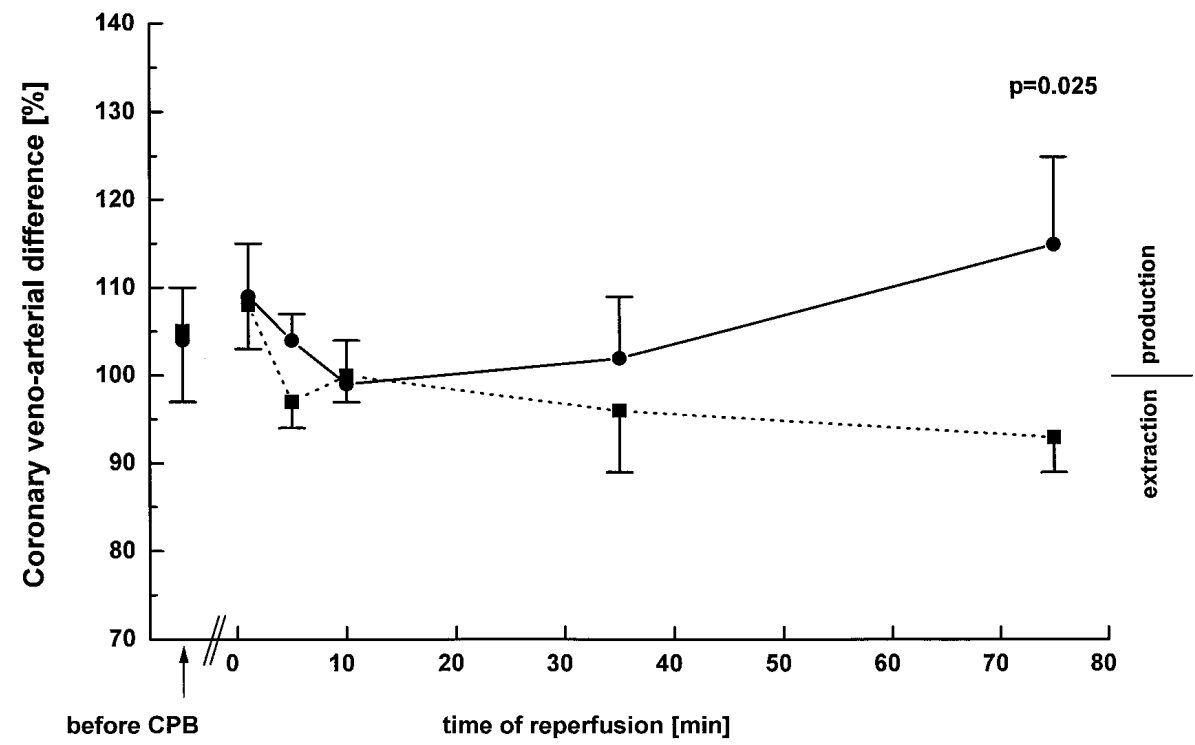

Fig 2. Coronary venoarterial difference of TNF- $\alpha$ levels, with the respective arterial value taken as $100 \%$. Values are means, and bars represent SEM ( $\mathrm{n}=15$ for each group; filled circles, placebo group patients; filled squares, SNP group patients). When differences between groups were significant, the respective levels are written on top of the symbols.

mal left ventricular function ${ }^{6}$ and again in the present study. In patients undergoing PTCA without myocardial infarction, no such cytokine release was observed. ${ }^{2}$ Thus the cardiac production of cytokines seems to be a phenomenon occurring in cases of severe ischemia and reperfusion.

In the present work the significant production of IL6 observed in control patients during early reperfusion 


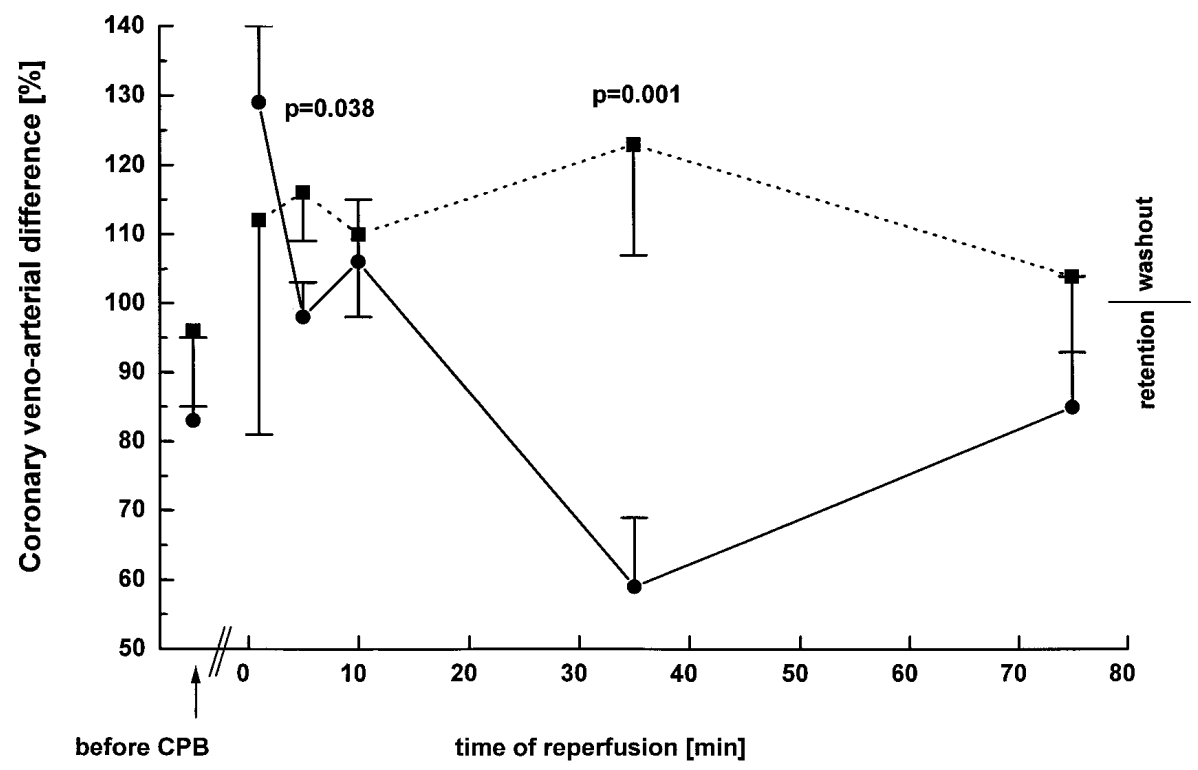

Fig 3. Coronary venoarterial difference of CD11b-positive monocytes, with the respective arterial value taken as $100 \%$. Symbols are means, and bars represent SEM ( $\mathrm{n}=15$ for each group; filled circles, placebo group patients; filled squares, SNP group patients). When differences between groups were significant, the respective levels are written on top of the symbols.

was mitigated by SNP. TNF- $\alpha$ release into coronary venous blood was greatest after 75 minutes of reperfusion in patients receiving placebo and was prevented in SNP-treated patients. The overall relatively small production of TNF- $\alpha$ observed here may well be due to the routine application of aprotinin. ${ }^{8}$ Cytokines have been reported to exert negative inotropic effects. ${ }^{7}$ The mitigation of such an influence might have been particularly beneficial in patients with compromised left ventricular function, as studied here. However, as the data on inotropic support show, there was no benefit with respect to the need for catecholamines under the application of SNP. In both groups the early postoperative course was relatively uneventful (no intra-aortic balloon pump and no low output syndrome), and therefore a potential functional benefit within this time period might have been obscured.

On the basis of actual cell counts, performed in a previous study of our group, ${ }^{1}$ the appearance of more activated leukocytes and platelets in the coronary venous blood indicates their washout, whereas a decline of activated cells is a sign of their retention in the coronary bed. Retained and activated leukocytes may be responsible for the release of cytokines. ${ }^{9}$ In recent studies on the inflammatory response of the lungs of patients undergoing CABG, significant cellular retention and subsequent cytokine release were demonstrat- ed. ${ }^{10,11}$ Retention is an indicator for interaction between the endothelium and the respective cells. The endothelium is activated during reperfusion, ${ }^{1}$ thus making the retention of activated cells more probable. The diminished retention and even washout in the case of SNP-treated patients observed in the present study may be interpreted as being cardioprotective because the related sequelae, such as chemotactic activation, transmigration, and burst reaction, may be prevented.

Marked activation of platelets during CPB has been described. ${ }^{12}$ The retention of activated platelets during coronary passage is a risk factor for restenosis. ${ }^{13}$ In the present study the influence of SNP on the interaction of activated platelets and the coronary endothelium led to a washout instead of retention of CD62-positive (Pselectin) platelets in patients receiving SNP. Activated blood platelets express the adhesion molecule CD41 (GpIIb/IIIa) on the membrane surface. This molecule (among others) allows platelets to coaggregate with the larger leukocytes, especially monocytes and PMNs. Again, washout of CD41-positive monocytes and PMNs in the present study indicates less interaction between the coronary vascular endothelium and platelet-leukocyte coaggregates under the application of SNP.

Surprisingly, no effects on the transmyocardial difference of quantitative cell counts were observed, 


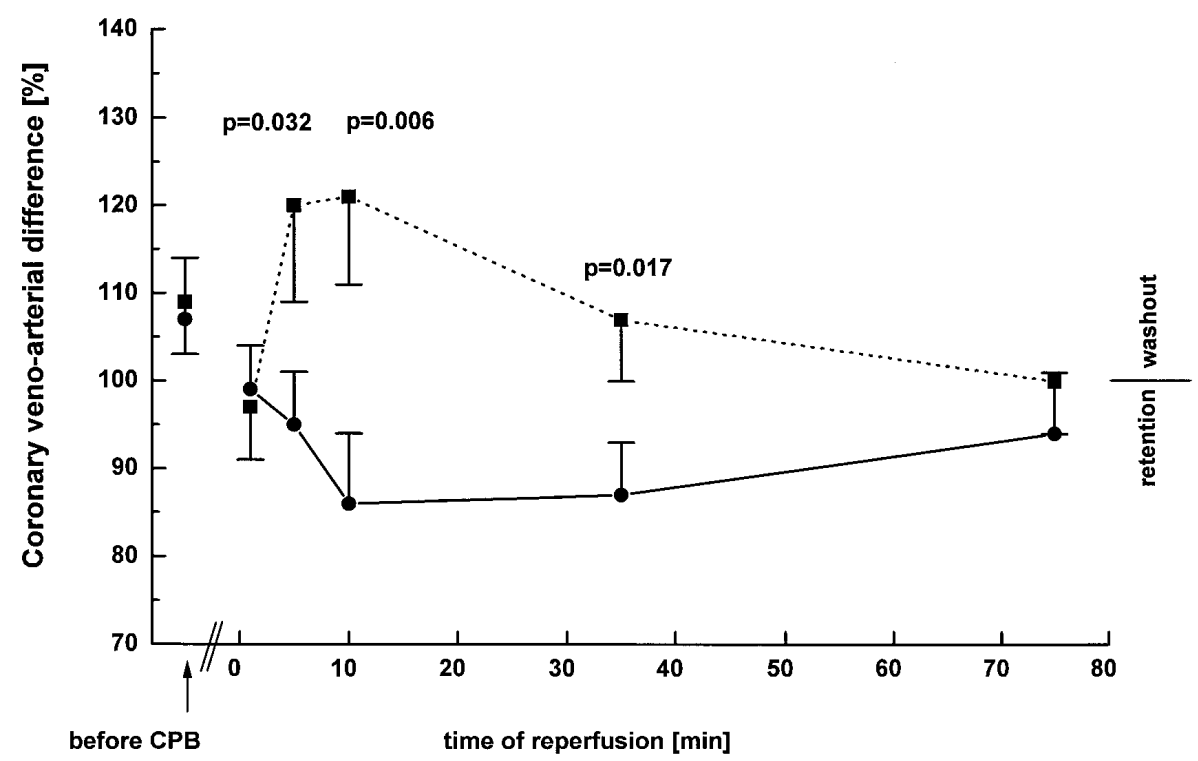

Fig 4. Coronary venoarterial difference of CD41-positive monocytes, with the respective arterial value taken as $100 \%$. Values are means, and bars represent SEM ( $\mathrm{n}=15$ each group; filled circles, placebo group patients; filled squares, SNP group patients). When differences between groups were significant, the respective levels are written on top of the symbols.

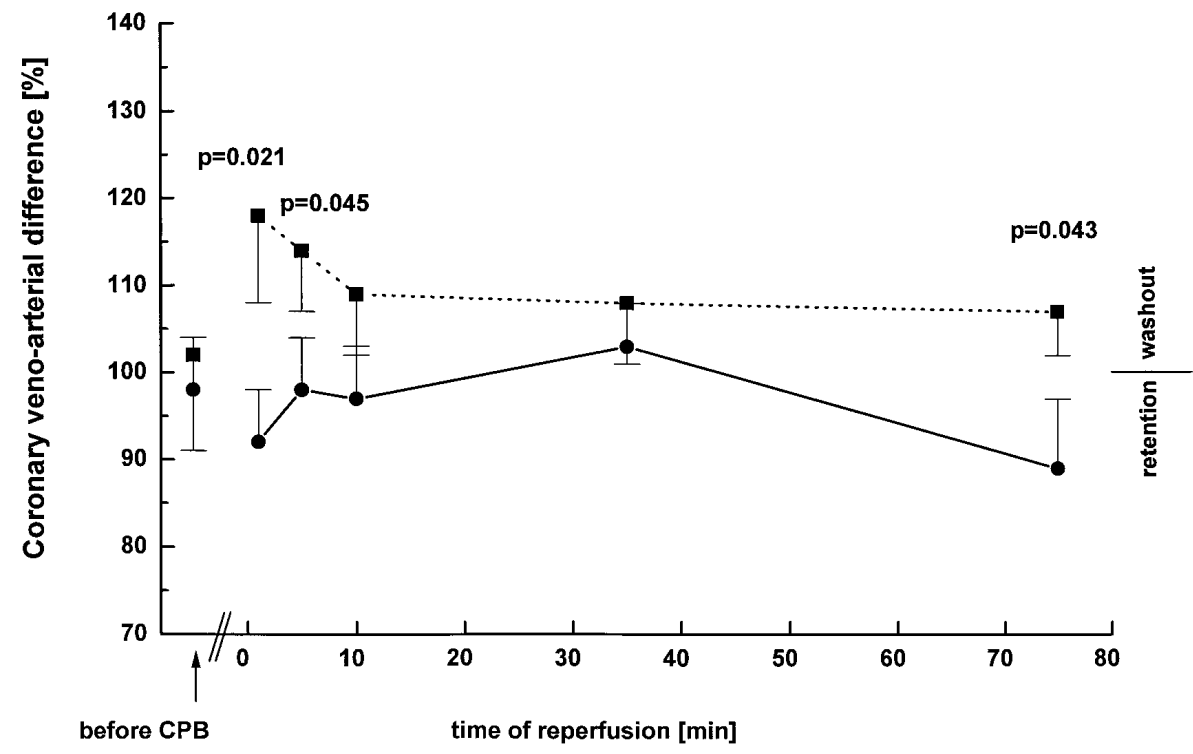

Fig 5. Coronary venoarterial difference of CD41-positive PMNs, with the respective arterial value taken as $100 \%$. Values are means, and bars represent SEM ( $\mathrm{n}=15$ for each group; filled circles, placebo group patients; filled squares, SNP group patients). When differences between groups were significant, the respective levels are written on top of the symbols.

which is in contrast to the pulmonary data in patients undergoing $\mathrm{CABG}$, in whom significant quantitative cellular retention is observed. ${ }^{10}$

Timing of intervention and the involved mechanism. The optimal timing of intervention with a donor of NO is unclear in the literature. Inhibition of the production of endogenous $\mathrm{NO}$ was found to be greatest starting with postischemic reperfusion. ${ }^{14}$ On the other hand, it was reported that recovery of the reperfused heart was greatest when NO was supplemented before 


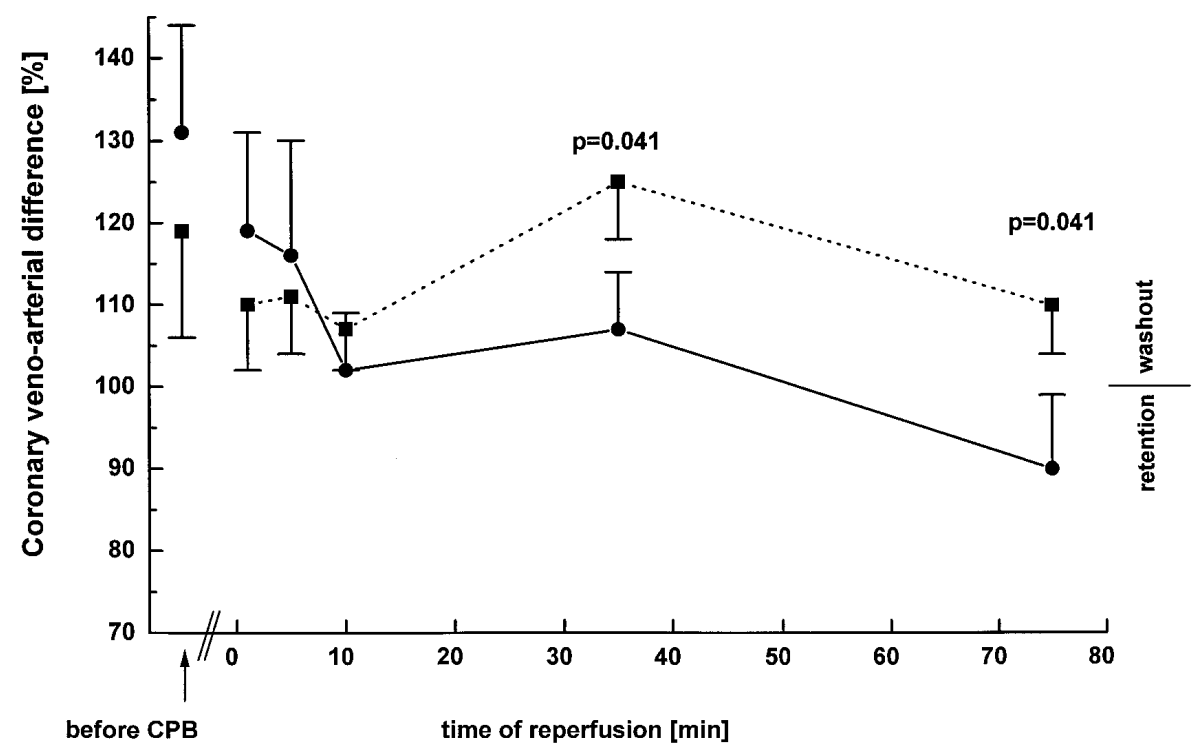

Fig 6. Coronary venoarterial ratio of CD62-positive platelets, with the respective arterial value taken as $100 \%$. Values are means, and bars represent SEM ( $\mathrm{n}=15$ for each group; filled circles, placebo group patients; filled squares, SNP group patients). When differences between groups were significant, the respective levels are written on top of the symbols.

cardioplegia and that the application during reperfusion may even be detrimental. ${ }^{15}$ In our own experience, on the basis of animal studies, the best results were obtained with the short-term postischemic application of an $\mathrm{NO}$ donor. $^{3}$ In the present study the duration of application was chosen to be 60 minutes instead of 20 minutes, as in a previous study. ${ }^{6}$ The reason was to prolong the previously observed anti-inflammatory effects. In patients with normal left ventricular function investigated in the previous study, the influence of SNP on the transcardiac production of cytokines and on the retention of activated leukocytes and platelets had, in fact, been weaker. ${ }^{6}$ However, the results of the 2 studies can only be compared with caution because (1) bubble oxygenators were used in the previous study and membrane oxygenators were used in the present study and (2) the administration of SNP was prolonged from 20 to 60 minutes.

On the one hand, free radical generation has been reported to be more pronounced ${ }^{16}$ and extravascular lung water has been reported to be increased ${ }^{17}$ in the case of bubble oxygenators. On the other hand, a large prospective study found no difference in duration of ventilation, intrapulmonary shunting, or hospital mortality rates between patients undergoing operations with bubble oxygenators and those undergoing operations with membrane oxygenators. ${ }^{18}$

An inhibiting effect on systemic complement activation by SNP infusion was observed in a study per- formed in children undergoing cardiac operations for congenital malformations. ${ }^{5}$ Complement activation was not measured in the present study but could occur subsequent to endothelial, leukocyte, and platelet activation. A radical scavenger effect of NO has been suggested from experimental studies. ${ }^{3,4}$ Although NO is known to be liberated from SNP, the exact mechanism of action in the present study remains unclear.

Taken together, the 60-minute administration of SNP used in the present study seemed to have a more pronounced mitigating effect on the production of proinflammatory stimuli and on the interaction between activated blood cells and the endothelium than the 20-minute application used before. ${ }^{6}$ Considering that different types of oxygenators were used, the fact that preoperative left ventricular function was compromised may have had a sensitizing influence. A number of studies have shown anti-inflammatory effects for different agents. The physiologic significance of the increased levels of proinflammatory substances in various situations of ischemia and reperfusion and their reduction by anti-inflammatory agents remains to be determined. Thus far, a functional benefit could not be demonstrated by any of the interventions.

In conclusion, after declamping of the aorta in patients undergoing CABG, SNP infusion reduces the transcardiac release of several proinflammatory stimuli. This is accompanied by a washout of activated leukocytes, platelets, and coaggregates of these 2 cell 
types. The latter indicates less interaction between the coronary endothelium and the respective activated cells, which should mitigate postoperative reperfusion complications.

We thank Ms M. Regensburger, Mrs E. Musiol, Ms K. Löschenkohl, and Ms M. Breilich for their excellent technical assistance.

\section{REFERENCES}

1. Zahler S, Massoudy P, Becker B, Hartl H, Hähnel C, Meisner H. Acute cardiac inflammatory responses to postischemic reperfusion during cardiopulmonary bypass. Cardiovasc Res 1999;41:722-30.

2. Neumann FJ, Ott I, Gawaz M, et al. Cardiac release of cytokines and inflammatory responses in acute myocardial infarction. Circulation 1995;92:748-55

3. Massoudy P, Becker BF, Gerlach E. Nitric oxide accounts for postischemic cardioprotection resulting from angiotensin-converting enzyme inhibition: indirect evidence for a radical scavenger effect in isolated guinea pig heart. J Cardiovasc Pharmacol 1995;25:440-7.

4. Kupatt C, Zahler S, Seligmann C, Massoudy P, Becker BF, Gerlach E. Nitric oxide mitigates leukocyte adhesion and vascular leak after myocardial ischemia. J Mol Cell Cardiol 1996;28:643-54.

5. Seghaye MC, Duchateau J, Grabitz RG, et al. Effect of sodium nitroprusside on complement activation induced by cardiopulmonary bypass: a clinical and experimental study. J Thorac Cardiovasc Surg 1996;111:882-92.

6. Massoudy P, Zahler S, Barankay A, Becker BF, Richter JA, Meisner H. Sodium nitroprusside during coronary artery bypass grafting: evidence for an anti-inflammatory action. Ann Thorac Surg 1999;67:1059-64.

7. Sugishita K, Kinugawa K, Shimizu T, et al. Cellular basis for the acute inhibitory effects of IL- 6 and TNF $\alpha$ on excitation-contraction coupling. J Mol Cell Cardiol 1999;31:1457-67.

8. Hill GE, Alonso A, Spurzem JR, Stammers AH, Robbins RA.
Aprotinin and methylprednisolone equally blunt cardiopulmonary bypass-induced inflammation in humans. $\mathrm{J}$ Thorac Cardiovasc Surg 1995;110:1658-62.

9. Marie C, Muret J, Fitting C, Losser MR, Payen D, Cavaillon JM. Reduced ex-vivo interleukin- 8 production by neutrophils in septic and nonseptic systemic inflammatory response syndrome. Blood 1998;91:3439-46.

10. Massoudy P, Zahler S, Becker BF, et al. Significant leukocyte and platelet retention during pulmonary passage after declamping of the aorta in CABG patients. Eur J Med Res 1999;4:178-82.

11. Massoudy P, Zahler S, Barankay A, Braun SL, Becker BF, Meisner $\mathrm{H}$. Reperfusion of the lungs induces cytokine production and activation of leukocytes in CABG patients [abstract]. Br J Anaesth 1999;82(Suppl 2):A50.

12. Rinder C, Fitch J. Amplification of the inflammatory response: adhesion molecules associated with platelet/white cell responses. J Cardiovasc Pharmacol 1996;27(Suppl 1):S6-12.

13. Ishiwata $S$, Tukada $T$, Nakanishi S, Nishiyama S, Seki A. Postangioplasty restenosis: platelet activation and the coagulation-fibrinolysis system as possible factors in the pathogenesis of restenosis. Am Heart J 1997;133:387-92.

14. Sato H, Zhao ZQ, Jordan JE, et al. Basal nitric oxide expresses endogenous cardioprotection during reperfusion by inhibition of neutrophil-mediated damage after surgical revascularization. J Thorac Cardiovasc Surg 1997;113:399-409.

15. Engelman DT, Watanabe M, Maulik N, et al. Critical timing of nitric oxide supplementation in cardioplegic arrest and reperfusion. Circulation 1996;94(Suppl):II407-11.

16. Abe H, Kumagai K, Matsuda T, Tanabe T, Mori H. Effect of ulinastatin on the free radical during cardiopulmonary bypass. Masui 1995;44:1005-9.

17. Iha K, Koja K, Kuniyoshi Y, et al. Extravascular lung water in patients after cardiac surgery [in Japanese]. Nippon Kyobu Geka Gakkai Zasshi 1993;41:389-95.

18. Reeve WG, Ingram SM, Smith DC. Respiratory function after cardiopulmonary bypass: a comparison of bubble and membrane oxygenators. J Cardiothorac Vasc Anesth 1994;8:502-8. 